\title{
A good complaints system
}

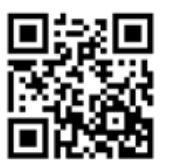

Healthcare professionals should accept that mistakes are an inevitable part of clinical practice. Despite what patients may think - and many expect doctors to be infallible, and the healthcare system to be flawless - medicine by its very nature is risky. Accepting that things will not always go to plan, and that patients will complain, should make doctors think about developing their own system for handling complaints.

While complaints often provide invaluable feedback on an individual's performance, they also offer dissatisfied patients an opportunity to air their views. Even if a complaint is without justification, a complaints system that works well will at least allow an individual to respond and give their account of the incident. This can help doctors avoid further, potentially more difficult, avenues of investigation such as by the Health Professions Council of South Africa (HPCSA) or a clinical negligence claim. Having a good-quality, standardised process for complaints handling, with well-trained staff in place, can help to avoid further anxiety for both parties. It is much better to embrace a complaint than to evade it. With understanding, empathy, action and honesty, a strong complaints handling system can benefit everyone involved.

Solutions to the growing number and severity of clinical negligence claims come in various forms. A key step in avoiding litigation is understanding what dissatisfied patients actually want following an adverse outcome. One way of doing this may be to establish a strong and comprehensive complaints system.

While a healthcare professional's natural inclination when an adverse outcome occurs may be to hide, having a good complaints system in place can offer benefits that prevent an individual from facing criticism. Ignoring complaints or failing to provide the means for patients to complain may simply force them down alternative routes, either by making a complaint to the HPCSA or by instructing an attorney to begin a claim.

\section{Why do patients sue?}

To help support the argument for a good complaints system, it is important to understand why patients are aggrieved with their care, and what leads them to sue. While financial compensation is an understandable goal in cases of severe injuries, most other patients primarily want answers, explanations and reassurance. They often seek a sense of closure that is undoubtedly provided by a full and honest clarification of what happened and why it went wrong. This is usually accompanied by a sincere apology, which in itself is not an admission of liability. In many cases, patients may only want acknowledgement that a complication has occurred, and there is widespread literature supporting this view. A study from Taiwan found that an emotional need for comfort and the sense of 'being cared for' was a key factor for patients and their families suing doctors, ${ }^{[1]}$ while a study published in The Lancet found that 'Patients taking legal action wanted greater honesty, an appreciation of the severity of the trauma they had suffered, and assurances that lessons had been learnt from their experiences.' The study surveyed 227 patients and their families who had instructed clinical negligence solicitors. ${ }^{[2]}$

\section{Alternative options}

Without the opportunity to make a complaint, a dissatisfied patient is likely to turn to other options. Patients may look to take the easiest route to expressing their dissatisfaction, but the options available to them are anything but easy: usually, these are either reporting a doctor to the HPCSA or instructing an attorney to make a claim for clinical negligence. Both processes are daunting in terms of the considerable length of time they can take, with the added emotional stress this brings for both doctor and patient. Neither is in the best interests of the doctor, nor will either option really give the patient the closure they want, unless their aim is obtaining financial compensation for a severe injury.

The HPCSA is bound by statute to investigate a complaint in the context of professional misconduct - it is not investigating the complaint, it is investigating a doctor's fitness to practise. Similarly, a law firm's interest is not in resolving a complaint - its interest is in terms of redress, and the focus will therefore be on establishing negligence. Yet many complaints have nothing to do with negligence. Neither approach is the quickest or the most satisfying route to resolution for either doctor or patient.

\section{What makes a good complaints system?}

When something goes wrong, the ensuing patient dissatisfaction has to be managed. The best way to do this is through a feedback system that allows the patient to deal with their dissatisfaction 'in-house', between themselves and the provider. ${ }^{[1]}$

A good complaints system:

- Understands the patient's dissatisfaction

- Sees the options that will resolve matters for the patient

- Tries to seek joint resolution.

A good complaints system is also able to suitably record and respond to a complaint in a way that demonstrates learning and improvement a learning culture rather than an adversarial culture. This allows for incidents to be reported as adverse outcomes and for the development of strategies to prevent similar errors occurring in the future.

Patients should be given the opportunity to voice their side of the story, to talk through the impact the incident has had on them, and to get the closure they need. A good system should also give healthcare professionals the chance to explain their side of the story in a nonadversarial environment, without fear of retribution, in an open and enquiring way.

There are a number of principles of excellent complaints handling: ${ }^{[3]}$ - Recognition of the complaint, so the patient feels acknowledged

- Understanding of why the patient is dissatisfied

- Showing empathy to demonstrate that the system and the doctor understands the emotional impact on the patient

- Effective action - a speed of response that is commensurate with the nature of the complaint; while a quick response can be satisfying for the patient, the complaint must be thoroughly investigated so that the patient can see that the response is not being made in haste, with speculation in place of insight

- Benefit for the patient - this could be an explanation, redress, commitment to get it right next time or reassurance that the problem will be rectified, to show that people have learned from the incident

- Honesty and transparency, so that the patient can see an open profession.

It may also be helpful for an impartial third party to intervene to mediate a complaint, if a patient is unable to resolve their concerns directly with a healthcare professional. ${ }^{[4]}$ Mediation can be a successful way to bring two parties together, and there are numerous professional organisations in South Africa that offer an impartial mediation service. 
For an example of a complaints system, read about COPIC on p. 436.

\section{Conclusion}

According to Johnston, ${ }^{[5]}$ an expert on customer service delivery, successful handling of complaints can build patient 'loyalty' and make them less likely to complain in the future. In this sense, patients are viewed as customers, and as most complaints are about minor service issues the relationship between doctor and patient does take on a customer service nature. More importantly, a good complaints handling service can give patients what they need after an adverse outcome in a much less painful manner than, for example, litigation. Surely this is in everyone's best interests?

\section{Graham Howarth}

Head of Medical Services in Africa, Medical Protection Society, Victoria House, 2 Victoria Place, Leeds, UK graham.howarth@mps.org.uk

\section{John Tiernan}

Executive Director, Member Engagement Division, Medical Protection Society, Victoria House, 2 Victoria Place, Leeds, UK
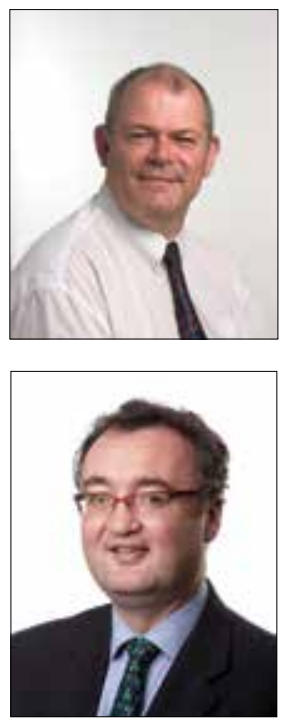

1. Chiu YC. What drives patients to sue doctors? The role of cultural factors in the pursuit of malpractice claims in Taiwan. Soc Sci Med 2010;71(4):702-707. [http://dx.doi.org/10.1016/j. socscimed.2010.04.040

2. Vincent C, Young M, Phillips A. Why do people sue doctors - a study of patients and relatives taking legal action. Lancet 1994;343(8913):1609-1613. [http://dx.doi.org/10.1016/S01406736(94)93062-7]

3. Tiernan J. The importance of complaints handling. Hong Kong Dent J 2006;3 (2):134-136.

4. Walters J. Mediation - an alternative to litigation in medical malpractice. S Afr Med J 2014;104(11):717718. [http://dx.doi.org/10.7196/SAMJ.8851]

5. Johnston R. Service failure and recovery: Impact, attributes and process. Advances in Services Marketing and Management: Research and Practice 1995;4:211-228.

S Afr Med J 2015;105(6):425-426. DOI:10.7196/SAMJ.9358

\section{Gareth Gillespie}

Communications Manager, Medical

Protection Society, Victoria House,

2 Victoria Place, Leeds, UK

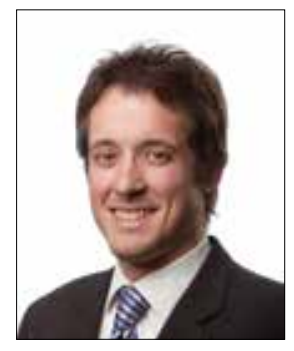

\section{Pieter Carstens}

Head of the Department of Public Law, Faculty of Law, University of Pretoria, South Africa

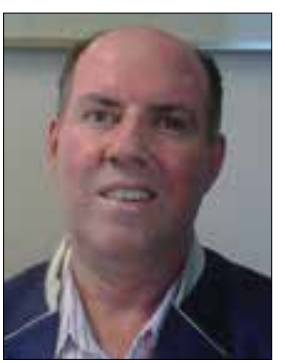

\section{This month in the SAMJ ...}

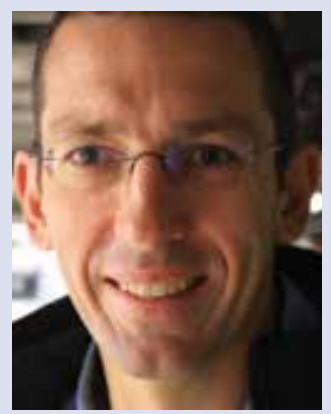

Bruce Biccard ${ }^{* \dagger}$ is an anaesthesiologist and Honorary Associate Professor at the University of KwaZulu-Natal. His principal area of practice is vascular anaesthesia, and his research interests include the epidemiology of cardiovascular disease, perioperative cardiovascular risk prediction and risk modification, perioperative medicine and surgical outcomes. He is an active member of the Perioperative Research Group, Department of Anaesthesiology and Critical Care, Nelson R Mandela School of Medicine, where national and international collaborative perioperative outcomes research is conducted. He is an active proponent of collaborative research to improve public health.

*Biccard BM, Madiba TE, on behalf of the Perioperative Research Group. The South African Surgical Outcomes Study: A 7-day prospective observational cohort study. S Afr Med J 2015;105(6):465-475. [http://dx.doi.org/10.7196/SAMJ.9435]

Moodley Y, Biccard BM. The impact of acute preoperative beta-blockade on perioperative cardiac morbidity and all-cause mortality in hypertensive South African vascular surgery patients. S Afr Med J 2015;105(6):476-479. [http://dx.doi.org/10.7196/SAMJ.8856]

Daniel Fiandeiro,* specialist in emergency medicine, is a graduate of the University of the Witwatersrand medical school. Currently working at Lister Hospital in the UK, he is an ATLS and level one ultrasound instructor with diplomas in emergency care and anaesthetics. He has a special interest in trauma and the prehospital and emergency care of burns.

*Fiandeiro D, Govindsamy J, Maharaj RC. Prehospital cooling of severe burns: Experience of the Emergency Department at Edendale Hospital, KwaZulu-Natal, South Africa. S Afr Med J 2015;105(6):457-460. [http://dx.doi.org/10.7196/SAMJ.8705]

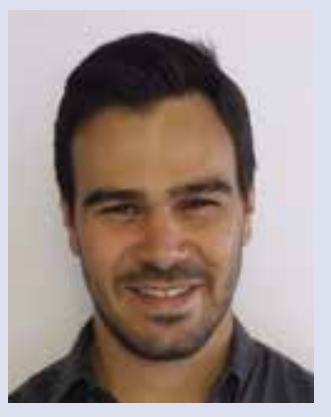

